\title{
The effectiveness of the TAX 327 nomogram in predicting overall survival in Chinese patients with metastatic castration-resistant prostate cancer
}

\author{
Xiao-Jie Bian ${ }^{1,2, *}$, Yao Zhu ${ }^{1,2, *}$, Yi-Jun Shen ${ }^{1,2}$, Jin-You Wang ${ }^{1,2}$, Chun-Guang Ma ${ }^{1,2}$, Hai-Liang Zhang ${ }^{1,2}$, \\ Bo Dai ${ }^{1,2}$, Shi-Lin Zhang ${ }^{1,2}$, Xu-Dong Yao ${ }^{1,2}$ and Ding-Wei Ye ${ }^{1,2}$
}

Based on the results of TAX 327, a nomogram was developed to predict the overall survival of metastatic castration-resistant prostate cancer (mCRPC) after first-line chemotherapy. The nomogram, however, has not been validated in an independent dataset, especially in a series out of clinical trials. Thus, the objective of the current study was to validate the TAX 327 nomogram in a community setting in China. A total of 146 patients with MCRPC who received first-line chemotherapy (docetaxel or mitoxantrone) were identified. Because clinical trials are limited in mainland China, those patients did not receive investigational treatment after the failure of first-line chemotherapy. The predicted overall survival rate was calculated from the TAX 327 nomogram. The validity of the model was assessed with discrimination, calibration and decision curve analysis. The median survival of the cohort was 21 months (docetaxel) and 19 months (mitoxantrone) at last follow-up. The predictive c-index of the TAX 327 nomogram was 0.66 (95\% Cl: $0.54-0.70)$. The calibration plot demonstrated that the 2-year survival rate was underestimated by the nomogram. Decision curve analysis showed a net benefit of the nomogram at a threshold probability greater than $30 \%$. In conclusion, the present validation study did not confirm the predictive value of the TAX 327 nomogram in a contemporary community series of men in China, and further studies with a large sample size to develop or validate nomograms for predicting survival and selecting therapies in advanced prostate cancer are necessary. Asian Journal of Andrology (2013) 15, 679-684; doi:10.1038/aja.2013.52; published online 1 July 2013

Keywords: castration-resistant; chemotherapy; nomogram; prostate cancer; validation studies

\section{INTRODUCTION}

Prostate cancer $(\mathrm{PCa})$ is the most common cancer and the second leading cause of cancer death in men, with an expected 28170 deaths in 2012 in the United States (American Cancer Society, 2012). Until 2007, the incidence and mortality rates of PCa were lower in mainland China than in Western countries. However, PCa-specific mortality has continued to rise. ${ }^{1}$ Currently, advanced or metastatic prostate cancer remains incurable, and androgen ablation could promise men with this disease a median progression-free time of 2 years. ${ }^{2}$ The duration from the first documented prostate-specific antigen (PSA) rise in the nonmetastatic castrate state to the terminal phase has been further extended to approximately 5 years. However, patients with progressive metastatic (radiographic detected) castration-resistant prostate cancer (mCRPC) have a worse prognosis, which is associated with a shorter survival time. ${ }^{3}$

The development of docetaxel-based chemotherapy has led to a milestone for the treatment of mCRPC, with an increased overall survival ranging from 17 to 21 months. ${ }^{4-6}$ Although there are emerging treatment options for patients with $\mathrm{mCRPC}^{7}$ docetaxel is still the first-line treatment in mainland China. In patients in the mCRPC stage, there is great interest in more accurately identifying patients who will benefit from chemotherapy. There are several factors that are suggested to correlate with overall survival, such as baseline PSA, lactate dehydrogenase, visceral disease, alkaline phosphatase, PSA doubling time, Gleason score, baseline pain status and cycles of chemotherapy. ${ }^{6,8-11}$ Those predictors of overall survival have been explored in both univariate and multivariate analysis in different models. The interplay between those factors is not linear, and the predictive accuracy is varied. To optimize the efficiency of these parameters, a nomogram, classification and regression tree analyses and risk group stratification models have been constructed based on these parameters. Of these models, nomograms present higher accuracy and have better performance characteristics than other alternatives. ${ }^{12}$

Some nomograms have been developed for phase III clinical trials evaluating cytotoxic chemotherapy to stratify risk groups. ${ }^{13,14}$ However, the patients included did not receive docetaxel-based therapy. Armstrong et al developed a novel nomogram that predicted the 1-, 2- and 5-year survival of mCRPC patients treated with docetaxelbased chemotherapy rooted in the TAX327 database. When adding clinical features, the model showed an area under the curve of 0.69 in the development cohort. ${ }^{15}$

${ }^{1}$ Department of Urology, Fudan University Shanghai Cancer Center, Shanghai 200032, China and ${ }^{2}$ Department of Oncology, Shanghai Medical College, Fudan University Shanghai 200032, China

* These authors contributed equally to this work.

Correspondence: Dr DW Ye (dwye.shca@gmail.com)

Received: 20 December 2012; Revised: 6 February 2013; Accepted: 30 March 2013; Published online: 1 July 2013 
The challenge of nomograms is that they are frequently based on a retrospective analysis with a certain group of patients. Before these encouraging findings can be used in routine clinical practice, they must be validated in an external unrelated cohort. Thus, we assessed the predictive ability of the established nomogram in a relatively small Chinese cohort of mCRPC patients after first-line chemotherapy with long-term follow-up.

\section{MATERIALS AND METHODS}

\section{Study population}

Between 2006 and 2011, a patient cohort with evidence of mCRPC from the Department of Urology at Fudan University Shanghai Cancer Centre was used to evaluate the validity of the TAX327 nomogram. The patients were administered docetaxel plus prednisone every 3 weeks or mitoxantrone plus prednisone every 3 weeks. Briefly, men were eligible if they had documented metastatic prostatic adenocarcinoma in the face of castrate levels of serum testosterone $\left(<50 \mathrm{ng} \mathrm{ml}^{-1}\right)$ and evidence of progression, as defined by clinically or radiographically measurable disease or increasing serum levels of PSA on three consecutive measurements obtained at least 1 week apart. Because clinical trials are limited in mainland China, those patients did not receive investigational treatment after the failure of first-line chemotherapy. The main end point of this analysis was overall survival, as defined by the time from enrolment to death. Patients who were alive as of the cutoff date were administratively censored, whereas patients lost to follow-up were censored at the time of the last follow-up. An approved and signed Institutional Review Board informed consent form was obtained from all participants.

\section{Nomogram}

The TAX327 nomogram (Figure 1) was originally developed and validated internally to predict 1-, 2- and 5-year cancer-specific survival using bootstrap methods with a concordance index (c-index) of 0.69. The variables required for the nomogram were collected, and detailed coefficients of each parameter were provided by Dr Armstrong and his college for validation.

\section{Statistical analysis}

Cox proportional hazards assumptions were measured with Schoenfeld residual analysis for univariate and multivariate analysis at a level of significance of 0.05 . Comparisons of baseline characteristics were performed using $95 \%$ confidence intervals (95\% CIs) and $t$-test statistical analysis.

To validate the nomogram externally, the c-index was estimated to calculate an unbiased measure of the predictive ability to discriminate among patients. In a binary outcome, the c-index is analogous to the area under a receiver-operating characteristic curve, which plots the sensitivity (true positive rate) against 1-specificity (false positive rate) for consecutive cutoffs to measure the discrimination ability of the models. ${ }^{16-18}$ Furthermore, the area under the curve $95 \%$ CI of the cindex value, calculated by bootstrapping using 200 resamples, was used to determine discrimination ability. Moreover, we constructed calibration plots of the TAX327 nomogram in which the $x$-axis represented the predicted probability, and the $y$-axis represented the observed probability of 1 - and 2-year survival. Clinical usefulness was assessed by decision curve analysis to estimate a net benefit for prediction models by summing the benefits and deducting the harms. It measures the probability of concordance between predicted receiver-operating characteristic curves that consider the full range

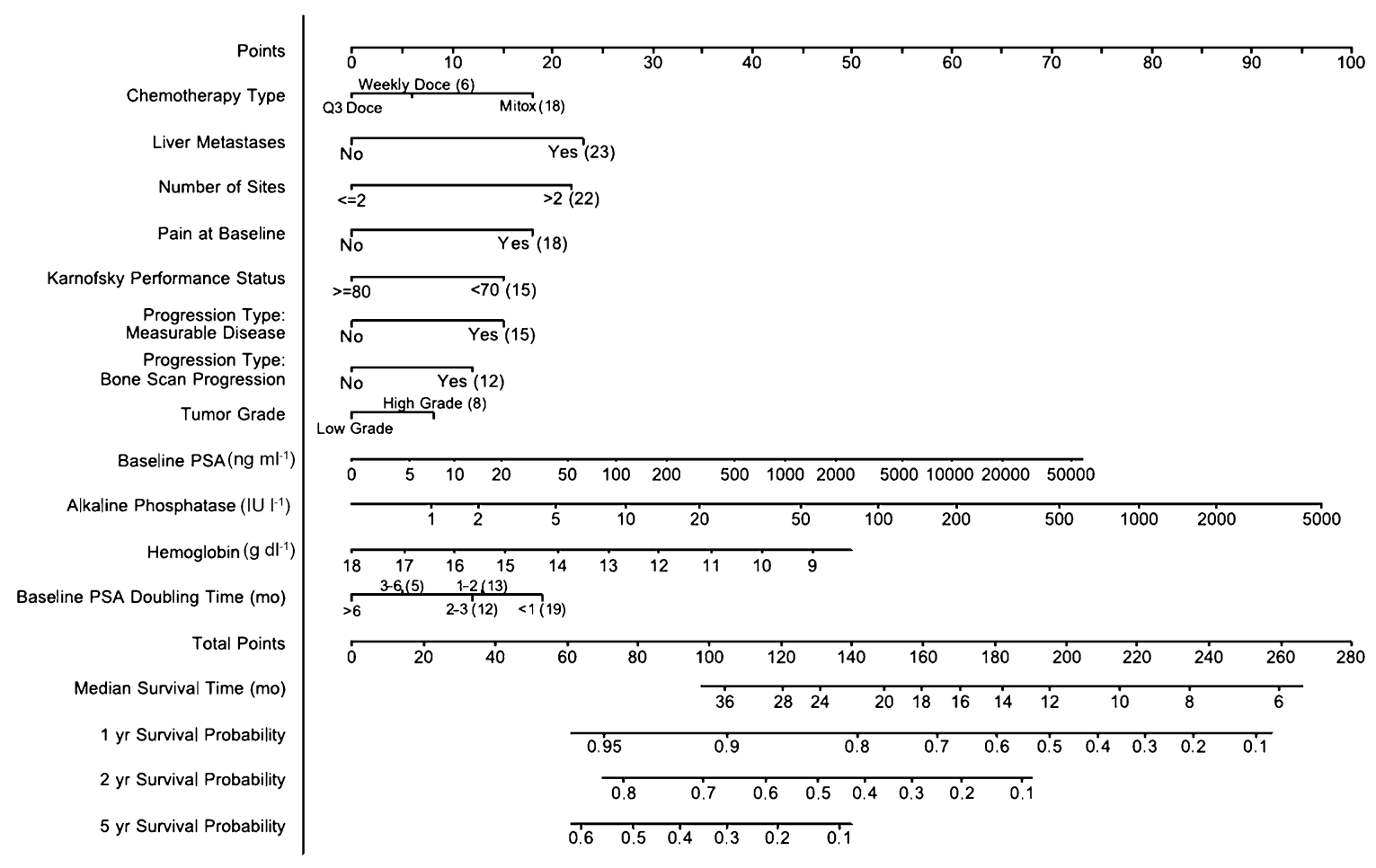

Figure 1 Nomogram for estimating the 1-, 2- and 5-year cancer-specific survival of mCRPC patients treated with Chemotherapy. To use the nomogram, determine the points assigned for each predictor variable on a scale of 0-100 and sum the points for each predictor. After that, locate this sum on the total points axis and draw a straight line downward from the total points axis to identify the predicted overall survival probabilities. 
Table 1 Baseline charactics of patients who underwent chemotherapy with a total number of 146

\begin{tabular}{|c|c|c|c|}
\hline & Docetaxel q3w & $\begin{array}{c}\text { Mitoxantrone } \\
q 3 w\end{array}$ & $\mathrm{p}$ \\
\hline No. randomized & 95 & 51 & \\
\hline Age & & & 0.461 \\
\hline Median (year) & 69 & 69 & \\
\hline Range (year) & $50-82$ & $50-82$ & \\
\hline$\geqslant 75$ year $(\%)$ & 25 & 16 & \\
\hline Gleason score (\%) & & & 0.884 \\
\hline$\leqslant 7$ & 19 & 29 & \\
\hline $8-10$ & 55 & 51 & \\
\hline Not available & 26 & 20 & \\
\hline Prior local therapy (\%) & 6 & 20 & 0.015 \\
\hline KPS score $\leqslant 70(\%)$ & 27 & 25 & 0.802 \\
\hline Pain at baseline(\%) & 53 & 63 & 0.242 \\
\hline Serum PSA & & & 0.200 \\
\hline Median (ng ml ${ }^{-1}$ ) & 90.31 & 100.86 & \\
\hline$\geqslant 20 \mathrm{ng} \mathrm{ml}^{-1}(\%)$ & 78 & 82 & \\
\hline \multicolumn{4}{|l|}{ Extent of disease (\%) } \\
\hline Bone metastases & 84 & 90 & 0.318 \\
\hline Visceral disease & 18 & 6 & 0.308 \\
\hline Measurable lesions & 36 & 27 & 0.308 \\
\hline \multicolumn{4}{|c|}{ Evidence of progression at entry (\%) } \\
\hline Bone scan & 15 & 8 & 0.100 \\
\hline Measurable lesions & 9 & 8 & 0.742 \\
\hline Increased PSA & 76 & 84 & 0.288 \\
\hline
\end{tabular}

Abbreviations: KPS, Karnofsky performance status; PSA, prostate-specific antigen.

of cutoffs rather than a single cutoff for a sensitivity/specificity pair. ${ }^{19,20}$ Statistical analyses were performed using R 2.13.0. All calculated $P$ values were two sided, and $P<0.05$ was considered statistically significant.

\section{RESULTS}

\section{Patient characteristics}

A total of 146 mCRPC patients who were treated with chemotherapy in 2006-2011 were included in the validation data set. Of these, 95 patients received docetaxel-based chemotherapy and 51 received mitoxantrone-based chemotherapy. The baseline characteristics are listed in Table 1. In the validation cohort, a higher rate of high Gleason Score, lower level of PSA and less progression of bone metastasis at baseline were identified.

In univariate Cox proportional hazards analysis (Table 2), performance status, baseline pain score and presence of visceral metastases showed a significant association with overall survival. The variables with the greatest risk of death included liver metastases (hazard ratio (HR): 2.87), baseline alkaline phosphatase (HR: 1.86), presence of visceral metastases (HR: 1.82) and metastatic sites involved (HR: 1.77). In multivariate Cox proportional hazards analysis, liver metastases (HR: 33.19; $P=0.001$ ) and significant baseline pain (HR: $1.47 ; P=0.002$ ) were screened as independent prognostic factors. Due to colinearity with liver metastasis and a weaker prognostic ability, sites of liver metastasis were dropped from the multivariate model. Additional factors were not entered due to lack of a significant prognostic effect.

\section{Survival}

There were 97 deaths among a total of 146 patients (66.4\%) and 2544 person-months of follow-up, with the longest time to death being 48 months during follow-up. The median survival of the cohort was 21 months and 19 months (docetaxel and mitoxantrone, respectively) at the last follow-up in June 2012. The cumulative 1-, 2- and 5-year disease-specific survival rates in the cohort were $75 \%, 32 \%$ and $6 \%$, respectively. The median survival period of all patients was 20.3 months (range: $4-48$ months).

\section{Evaluation of the predictive accuracy}

A total of 200 bootstrap resamples were used for external validation of the accuracy evaluation and to reduce overfit bias, and this gave a predictive accuracy of 0.66 (95\% CI: $0.54-0.70$ ) (Figure 2). The calibration plot of the TAX327 nomogram demonstrated that the predicted probability of cancer-specific survival after 1 year was consistent with the actual probability. However, the predicted probability of causespecific survival after 2 years was underestimated, as shown by the logistic calibration curve, which had important departures from ideal

Table 2 Univariate Cox proportional hazards analysis

\begin{tabular}{|c|c|c|c|}
\hline Variables & $\mathrm{n}$ & Univariate HR (95\% Cl) & $P$ \\
\hline Liver metastases (yes/no) & 146 & $2.87(0.89,9.20)$ & 0.076 \\
\hline No. sites involved (>2 vs. V2) & 146 & $1.77(0.47,6.68)$ & 0.400 \\
\hline Performance status at baseline ( $\leqslant 70$ vs. $\geqslant 80)$ & 146 & $0.95(0.93,0.96)$ & $<0.001$ \\
\hline Pain at baseline & 146 & $1.52(1.20,1.93)$ & 0.001 \\
\hline Multiple hotspots on bone scan (yes/no) & 146 & $0.77(0.97,1.03)$ & 0.767 \\
\hline Visceral metastases (yes/no) & 146 & $1.82(1.20,2.78)$ & 0.005 \\
\hline PSADT ( $<50$ days vs. $\geqslant 50$ days) & 146 & $1.03(0.69,1.53)$ & 0.902 \\
\hline \multicolumn{4}{|l|}{ Baseline PSA } \\
\hline$\geqslant 90 \mathrm{ng} \mathrm{ml}^{-1}$ (vs. $<90 \mathrm{ng} \mathrm{ml}^{-1}$ ) & 146 & $1.37(0.92,2.05)$ & 0.123 \\
\hline Per log unit rise in $\mathrm{ng} \mathrm{ml}^{-1}$ & & $1.34(1.00,1.79)$ & 0.050 \\
\hline Baseline ALP (per log unit rise in IU I ${ }^{-1}$ ) & 146 & $1.86(1.08,3.19)$ & 0.024 \\
\hline Age $(\geqslant 69$ years vs. $<69$ years $)$ & 146 & $1.16(0.78,1.73)$ & 0.457 \\
\hline Type of progression & 146 & & \\
\hline Measurable disease & & $1.17(0.62,2.18)$ & 0.634 \\
\hline Bone scan progression & & $1.12(0.65,1.92)$ & 0.693 \\
\hline Rising PSA only & & $0.88(0.55,1.41)$ & 0.596 \\
\hline Tumour grade (Gleason score $>7$ vs. $<7$ ) & 111 & $1.15(0.69,1.89)$ & 0.597 \\
\hline Prior radiotherapy (yes/no) & 146 & $1.53(0.83,2.82)$ & 0.172 \\
\hline Baseline haemoglobin (per unit decline, $\mathrm{g} \mathrm{dl}^{-1}$ ) & 146 & $0.99(0.98,1.00)$ & 0.035 \\
\hline Treatment group (docetaxel vs. mitoxantrone) & 146 & $1.50(0.99,2.26)$ & 0.056 \\
\hline
\end{tabular}

Abbreviations: ALP, alkaline phosphatase; PSA, prostate-specific antigen; PSADT, prostate-specific antigen doubling time. 
1-year

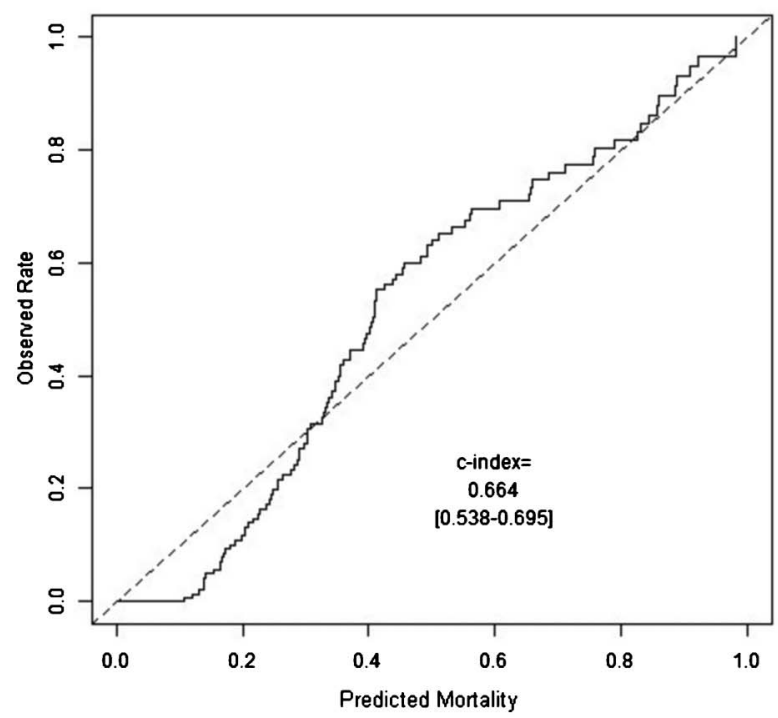

2-year

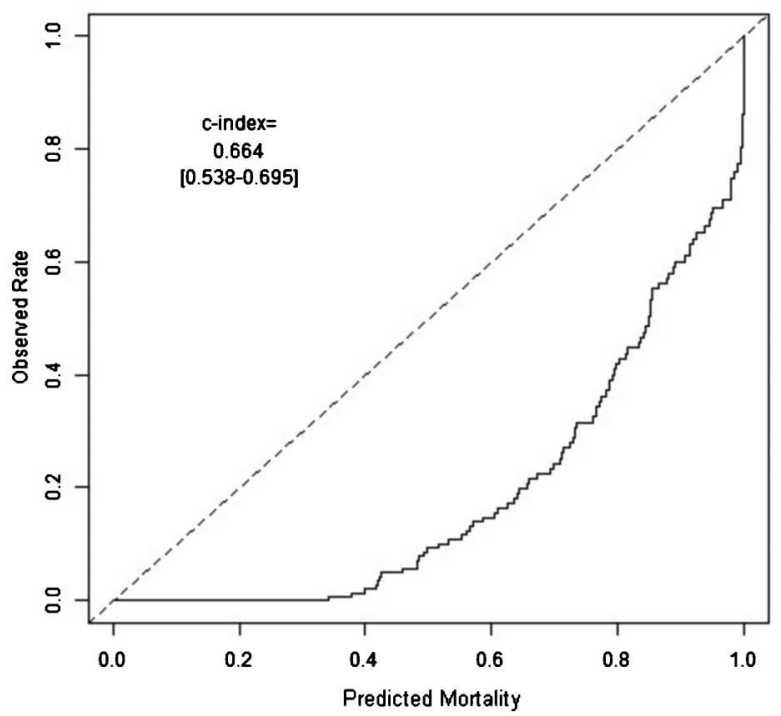

Figure 2 Calibration plot of 1- and 2-year survival rate of TAX327 nomogram in validation data set of 146 patients shows discrimination and calibration statistics.

prediction (Figure 3). Decision curve analysis showed a net benefit of the nomogram at a threshold probability greater than $30 \%$ (Figure 4).

\section{DISCUSSION}

Compared with post-docetaxel evaluation, life expectancy before docetaxel-based therapy always influences the decision making of both the clinician and patients. The prognostic nomogram was first developed by Armstrong et al to predict the survival rate among men with mCRPC after chemotherapy. ${ }^{15}$ In addition to traditional prognostic markers, this multivariate prognostic model, based on the TAX327 trial, included some novel prognostic variables, such as pre-treatment PSA kinetics, the presence of pain, number of metastatic sites and the type of disease progression at baseline. In their study, the area under the curve was 0.69 for predicting the 1-, 2- and 5-year survival. Univariate Cox proportional

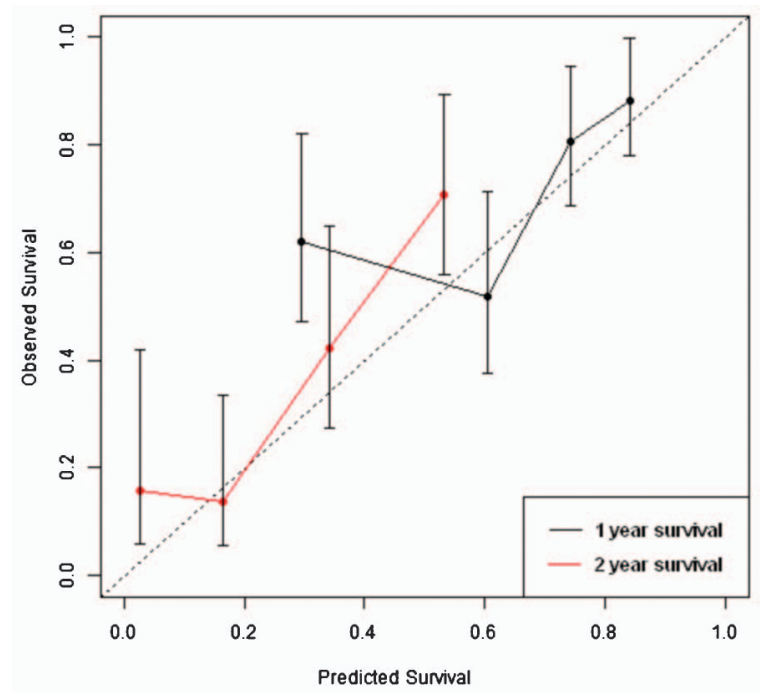

Figure 3 Calibration curve comparison between the 1- and 2-year survival in validation data set of 146 patients with 97 deaths. All the patients were divided into 4 groups with 36 patients each. hazards analysis confirmed prior reports and added validity to the Halabi nomogram. Compared with the classic Halabi nomogram, the median survival with TAX327 was longer than in the Halabi nomogram, in which the majority of patients were not treated with chemotherapeutic regimens, such as docetaxel or mitoxantrone with prednisone. ${ }^{10,13,15}$

Application of the novel TAX327 nomogram has been limited by the lack of external validation. In our study, we used a Chinese cohort of 146 patients to validate the predictive model, but we note that our ethnic population was limited, and not all patients were treated with docetaxel. Frustratingly, we did not confirm the ability of this predictive model to stratify the prognosis of patients after chemotherapy to predict the survival rate.

In the discrimination analysis, the estimated value of the c-index of our series was 0.66 (95\% CI: 0.54-0.70); thus, the nomogram did not discriminate perfectly among patients in the external validation sets using only nomogram score and the status of overall survival. However, this pre-chemotherapy nomogram allows for the standardisation of care and decision making if the predictive survival time is less than 1 year. Meanwhile, the goodness-of-fit test of the calibration plot showed that the nomogram model had a significantly good fit at 1 -year survival intervals, whereas the 2 -year survival rate, which was

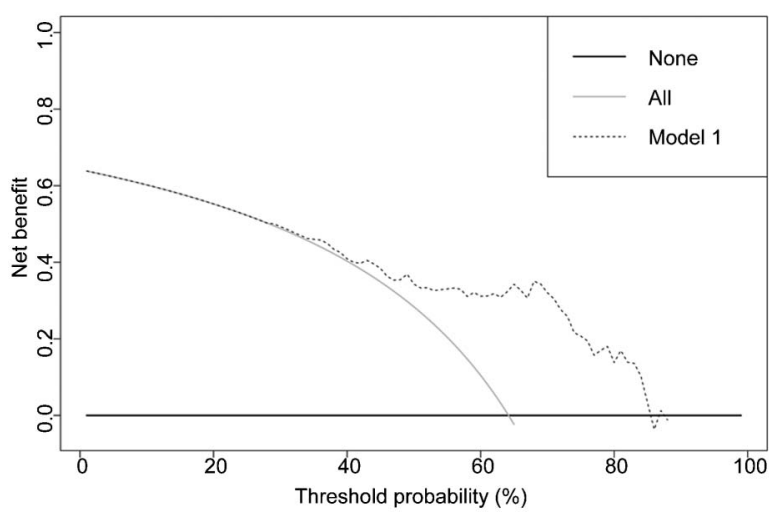

Figure 4 Decision curve of predicted probability net benefit using nomogram of Armstrong et al. in validation data set of 146 patients. 
investigated graphically using local regression nonparametric smoothing lines, was markedly underestimated. Therefore, the actual and predicted survival outcomes presented an imperfect agreement. In addition to discrimination and calibration, we further evaluated the clinical usefulness to facilitate decision making on the nomogram interventions. In decision curve analysis, the TAX327 nomogram showed a good net benefit and reduction at a threshold probability of $30 \%$ or greater. The effectiveness of the model is better than treating all patients indiscriminately with the condition that the decisionmaking risk is greater than $30 \%$.

With regard to the TAX327 nomogram, the achieved predictive accuracy of the nomogram in the validation cohort was not similar to the statistic in the original data set. Interestingly, survival in this off-study population was demonstrated to be worse than in the clinical trial. ${ }^{21}$ The reasons for this phenomenon are varied. First, the TAX327 nomogram was derived from a dataset that is now nearly 10 years old. Current clinical trials have illustrated the overall survival spectrum of standard 3-week docetaxel as 16.9-24.2 months in Western countries. ${ }^{22}$ In Asian countries, off-trial studies presented an overall survival ranging from 15.3 to 27.1 months. ${ }^{23-25}$ Second, although these novel agents (provenge, abiraterone and cabazitaxel) have not been introduced in mainland China in the past decade, improvement of the treatment environment, more regular follow-up schedules of patients and more positive feedback from caregivers could contribute to longer survival. ${ }^{21}$ Third, previous studies have predominantly been composed of Caucasian and European-American individuals, and the TAX327 study has not been prospectively validated in Asian populations. As proved before, the mortality rates of $\mathrm{PCa}$ are lower in China, and the heterogeneity of prostate cancer in a different population may be another reason contributing to slow progression. ${ }^{1}$ Fourth, earlier treatment with chemotherapy, which could extend the survival time from the beginning of enrolment, would result in leading bias of this validation. Finally, the numeric range of the $95 \%$ CI for predictive survival rate is large because the validation was performed in a small Chinese community dataset, and the cohorts enrolled were retrospectively collected at a single tertiary centre. The sample size of the validation study is still far from perfect, and the results may have fluctuated due to selection bias. Subsequently, validation with larger cohort needs to be performed, initiating multicentre collaborations. Further modifications of our validation are still necessary.
We believe that this nomogram still plays a role, even in the dawn of an era with an entirely new portfolio of agents for mCRPC. First, the external validation of a nomogram is conducive to take full advantage of the previous valuable information and avoid the shortage of reestablishment of a nomogram based on small datasets, such as instability of the model and dissociation of screened variables. Second, the life expectancy of mCRPC largely depends on the therapeutic option, management of comorbidities and life quality. Thus, the nomogram may be helpful for treatment decision making in surrogates who are at high risk of short-term survival, to avoid toxicities of chemotherapy and to switch to alternative emerging therapeutic options. Additionally, for patients at low risk of short-term survival, after docetaxel chemotherapy, both abiraterone acetate and cabazitaxel are available. ${ }^{26}$ Because there are no head-to-head clinical trials to compare those drugs, both clinicians and patients are confused when choosing the most suitable agent. As a predictive model, the nomogram can inevitably help make treatment decisions to meet the demand of quality and expectancy of life and to encourage individual counselling and tailored decision making. Finally, this nomogram is still valuable because the speed of new agents listed on the market is slow in mainland China.

\section{CONCLUSION}

Our validation study did not confirm the predictive value of the TAX 327 nomogram in a contemporary Chinese community series. As the speed of new agents listed on the market is slow in mainland China, further studies with large sample sizes to develop or validate nomograms for predicting survival and selecting therapies in advanced prostate cancer are necessary.

\section{AUTHOR CONTRIBUTIONS}

$\mathrm{XJB}$ and YZ designed the study, analyzed and interpreted the clinical data, wrote and revised the manuscript. YJS collected most clinical and followed up data. DWY supervised the project and revised the manuscript. JYW, CGM, HLZ, BD, SLZ and XDY collected partial patients' clinical data and followed up with patients. All the authors vouch for the data and analysis, approved the final version and agreed to publish the manuscript.

\section{COMPETING FINANCIAL INTERESTS}

The authors declare that they have no financial or commercial interests related to this study.

Appendix 1 Detailed information for the coefficient of TAX327 nomogram

\begin{tabular}{|c|c|c|c|c|}
\hline Category & coefficient & $\operatorname{se}(\operatorname{coef})$ & $z$ & $\mathrm{p}$ \\
\hline Weekly docetaxel & 0.119 & 0.1108 & 1.07 & 2.82E-01 \\
\hline Mitoxantrone & 0.371 & 0.1081 & 3.43 & 5.98E-04 \\
\hline Liver metastasis & 0.48 & 0.2169 & 2.21 & 2.69E-02 \\
\hline No. metastatic sites $(>2$ or $\leqslant 2)$ & 0.468 & 0.1442 & 3.24 & $1.18 \mathrm{E}-03$ \\
\hline Pain at baseline & 0.382 & 0.0971 & 3.93 & 8.46E-05 \\
\hline Performance status & 0.304 & 0.1388 & 2.19 & 2.85E-02 \\
\hline Measureable disease progression & 0.31 & 0.1125 & 2.75 & 5.90E-03 \\
\hline Bone scan progression & 0.256 & 0.1003 & 2.55 & 1.08E-02 \\
\hline Tumor grade & 0.172 & 0.0926 & 1.86 & 6.29E-02 \\
\hline Baseline log PSA & 0.153 & 0.0313 & 4.87 & $1.11 \mathrm{E}-06$ \\
\hline Alkaline phosphatase & 0.238 & 0.0489 & 4.86 & 1.19E-06 \\
\hline Hemoglobin & -0.107 & 0.0355 & -3.01 & 2.64E-03 \\
\hline PSADT(1-2months) & -0.131 & 0.1307 & -1.00 & 3.16E-01 \\
\hline PSADT(2-3months) & -0.147 & 0.1457 & -1.01 & 3.11E-01 \\
\hline PSADT(3-6months) & -0.291 & 0.1613 & -1.80 & 7.13E-02 \\
\hline PSADT(>6months) & -0.392 & 0.2425 & -1.62 & $1.06 \mathrm{E}-01$ \\
\hline
\end{tabular}

Abbreviations: PSA, prostate-specific antigen; PSADT, prostate-specific antigen doubling time. 


\section{ACKNOWLEDGMENTS}

We thank Andrew J Armstrong from the Duke Cancer Institute and Elizabeth S Garrett-Mayer from the Medical University of South Carolina for providing detailed information on the TAX327 nomogram (Appendix 1) and helpful discussion. This study was supported in part by grants from the National Natural Science Foundation of China (No. 81272837) and Doctoral Exchange Program of Fudan University.

1 Han RQ, Wu M, Chen WQ, Zhang SW, Zhen RS. Analysis on incidence and mortality of prostate cancer in China during 2003-2007. China Cancer 2012; 21: 805-11.

2 Harris W, Mostaghel E, Nelson P, Montgomery B. Androgen deprivation therapy: progress in understanding mechanisms of resistance and optimizing androgen depletion. Nature clinical practice. Urology 2009; 6: 76-85.

3 Antonarakis E, Eisenberger M. Expanding treatment options for metastatic prostate cancer. N Engl J Med 2011; 364: 2055-8.

4 Petrylak DP, Tangen CM, Hussain MH, Lara PN, Jones JA et al. Docetaxel and estramustine compared with mitoxantrone and prednisone for advanced refractory prostate cancer. N Engl J Med 2004; 351: 1513-20.

5 Tannock IF, de Wit R, Berry WR, Horti J, Pluzanska A et al. Docetaxel plus prednisone or mitoxantrone plus prednisone for advanced prostate cancer. N Engl J Med 2004; 351: 1502-12.

6 Berthold D, Pond G, Soban F, de Wit R, Eisenberger M et al. Docetaxel plus prednisone or mitoxantrone plus prednisone for advanced prostate cancer: updated survival in the TAX 327 study. J Clin Oncol 2008; 26: 242-5.

7 Shore N, Mason M, de Reijke T. New developments in castrate-resistant prostate cancer. BJU Int 2012; 109 (Suppl 6): 22-32.

8 Daskivich T, Regan M, Oh W. Distinct prognostic role of prostate-specific antigen doubling time and velocity at emergence of androgen independence in patients treated with chemotherapy. Urology 2007; 70: 527-31.

9 Graff J, Beer T. Toward predictors of survival in castration-resistant prostate cancer. Cancer 2011; 117: 3882-4.

10 Kawahara T, Miyoshi Y, Sekiguchi Z, Sano F, Hayashi N et al. Risk factors for metastatic castration-resistant prostate cancer (CRPC) predict long-term treatment with docetaxel. PloS ONE 2012; 7: e48186.

11 Qu YY, Dai B, Kong YY, Ye DW, Yao XD et al. Prognostic factors in Chinese patients with metastatic castration-resistant prostate cancer treated with docetaxel-based chemotherapy. Asian J Androl 2013; 15: 110-5.

12 Chun FK, Karakiewicz PI, Briganti A, Walz J, Kattan MW et al. A critical appraisal of logistic regression-based nomograms, artificial neural networks, classification and regression-tree models, look-up tables and risk-group stratification models for prostate cancer. BJU Int 2007; 99: 794-800.

13 Halabi S, Small E, Kantoff P, Kattan M, Kaplan E et al. Prognostic model for predicting survival in men with hormone-refractory metastatic prostate cancer. J Clin Oncol 2003; 21: 1232-7.

14 Smaletz O, Scher H, Small E, Verbel D, McMillan A et al. Nomogram for overall survival of patients with progressive metastatic prostate cancer after castration. J Clin Oncol 2002; 20: 3972-82.

15 Armstrong AJ, Garrett-Mayer ES, Yang YC, de Wit R, Tannock IF et al. A contemporary prognostic nomogram for men with hormone-refractory metastatic prostate cancer: a TAX327 study analysis. Clin Cancer Res 2007; 13: 6396-403.

16 Steyerberg EW, Vickers AJ, Cook NR, Gerds T, Gonen M et al. Assessing the performance of prediction models: a framework for traditional and novel measures. Epidemiology 2010; 21 : 128-38.

17 Pepe M, Feng Z, Huang $Y$, Longton G, Prentice $R$ et al. Integrating the predictiveness of a marker with its performance as a classifier. Am J Epidemiol 2008; 167: 362-8.

18 Harrell FE, Lee KL, Mark DB. Multivariable prognostic models: issues in developing models, evaluating assumptions and adequacy, and measuring and reducing errors. Stat Med 1996; 15: 361-87.

19 Vickers AJ, Elkin EB. Decision curve analysis: a novel method for evaluating prediction models. Med Decis Making 2006; 26: 565-74.

20 Vickers AJ, Cronin AM, Elkin EB, Gonen M. Extensions to decision curve analysis, a novel method for evaluating diagnostic tests, prediction models and molecular markers. BMC Med Inform Decis Mak 2008; 8: 53.

21 Goyal J, Nuhn P, Huang P, Tyagi P, Oh D et al. The effect of clinical trial participation versus non-participation on overall survival in men receiving first-line docetaxelcontaining chemotherapy for metastatic castration-resistant prostate cancer. BJU Int 2012; 110: 575-82.

22 Serpa Neto A, Tobias-Machado M, Kaliks R, Wroclawski M, Pompeo AN et al. Ten years of docetaxel-based therapies in prostate adenocarcinoma: a systematic review and meta-analysis of 2244 patients in 12 randomized clinical trials. Clin Genitourin Cancer 2011; 9: 115-23.

23 Miyake H, Sakai I, Harada KI, Muramaki M, Fujisawa M. Significance of docetaxelbased chemotherapy as treatment for metastatic castration-resistant prostate cancer in Japanese men over 75 years old. Int Urol Nephrol 2012; 44: 1697-703.

24 Kita Y, Shimizu Y, Inoue T, Kamba T, Yoshimura K et al. Reduced-dose docetaxel for castration-resistant prostate cancer has no inferior impact on overall survival in Japanese patients. Int J Clin Oncol 2012; e-pub ahead of print 13 July 2012; doi:10.1007/s10147-012-0443-3.

25 Miura N, Numata K, Kusuhara Y, Shirato A, Hashine K et al. Docetaxel-prednisolone combination therapy for Japanese patients with hormone-refractory prostate cancer: a single institution experience. Jpn J Clin Oncol 2010; 40: 1092-8.

26 Mohler J, Armstrong A, Bahnson R, Boston B, Busby J et al. Prostate cancer, Version 3.2012: featured updates to the NCCN guidelines. JNCCN 2012; 10: 1081-7. 